\title{
Self-dual Ginzburg-Landau vortices in a disk
}

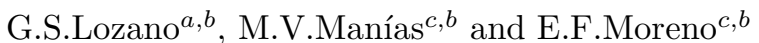 \\ ${ }^{a}$ Depto. de Física, FCEyN, Universidad de Buenos Aires, Pab I, Ciudad Univeristaria, Buenos Aires, Argentina. \\ ${ }^{b}$ Consejo Nacional de Investigaciones Científicas y Técnicas, Argentina. \\ ${ }^{c}$ Depto. de Física. Universidad Nacional de La Plata. CC 67, 1900 La Plata, Argentina.
}

(May 2000)

\begin{abstract}
We study the properties of the Ginzburg-Laundau model in the self-dual point for a twodimensional finite system . By a numerical calculation we analyze the solutions of the Euler-Lagrange equations for a cylindrically symmetric ansatz. We also study the self-dual equations for this case. We find that the minimal energy configurations are not given by the Bogomol'nyi equations but by solutions to the Euler Lagrange ones. With a simple approximation scheme we reproduce the result of the numerical calculation.
\end{abstract}

Pacs: 11.27.+d,74,74.20.De,74.60.Ec

Keywords: Vortices, Superconductivity, Ginzburg-Landau, Mesoscopics.

The study of vortex solutions in Ginzburg Landau (GL) theories has been the subject of continuous interest in different areas of condensed matter and high energy physics.

Static solutions in 2-dimensional infinite samples are characterized by the dimensionless GL parameter $\kappa$ which is defined as the ratio of the magnetic penetration length $\lambda$ and the coherence length $\zeta$. It has been known for several years that the GL model in the infinite plane possesses very special properties at $\kappa^{2}=1 / 2$. For instance the second order static equations of motion are equivalent to a set of much simpler first order equations referred as self-dual or Bogomol'nyi equations (BE) [1].

Although the existence of self-dual equations was first pointed out within the study of superconductors by Harden and Arp [2] most of the research on the subject has been done in connection with the role this type of equations play in High Energy Physics. Indeed, self-dual equations were introduced in this context independently by Bogomol'nyi and de Vega and Schaposnik [1] in their study of vortex solutions of the relativistic version of the GL model (Abelian Higgs model). Since then, the properties of the solutions, the connection with Supersymmetry, Topological Field Theories and Duality have been established not only for the Abelian Higgs model but also for related theories in different number of space time dimensions and non-Abelian gauge groups [3].

Very recently, Akkermans and Mallick have addressed the study of the GL model at the self-dual point for a 2dimensional disk of finite radius $R$ [ $[$. Their study is relevant for the case of vortices in mesoscopic systems, where the size of the sample is of the order of $\lambda$ and $\zeta$. As evidenced by recent experiments [5], the superconducting behavior of mesoscopic disks is radically different from that of the same material in the macroscopic regime [6]. An interesting question that arises is then to determine in which way size effects manifest at the self-dual point and reciprocally to analyze if the special properties that the model show at the self-dual point allow for a simpler interpretation of the experimental results

In this work we shall re-analyze the properties of the GL model for finite systems in the self-dual point. We will perform a numerical study of the equation of motion and explore the role played by the self-dual equations for this case. As a result of our analysis it will be shown that some of the approximations made in [4] are not correct. We shall in turn present a simple approximation scheme which correctly reproduces the numerical calculation.

The GL expression for the energy of a 2-dimensional sample $\Omega$ can be written as

$$
E=\int d^{2} x\left\{\frac{1}{16 \pi} F_{i j} F_{i j}+\frac{1}{2}\left|D_{i} \phi\right|^{2}+V(|\phi|)\right\}
$$

where $F_{i j}=\partial_{i} A_{j}-\partial_{j} A_{i}$, and $D_{i} \phi=\partial_{i} \phi-i q A_{i} \phi$ with $i=1,2$. Here $A_{i}$ denotes the electromagnetic vector potential, $\phi$ is a complex scalar field (order parameter) and $q$, the charge. Writing the potential as

$$
V(|\phi|)=\beta / 2\left(|\phi|^{2}-\eta^{2}\right)^{2},
$$

the penetration length and the coherence length are $\lambda^{2}=1 /\left(4 \pi q^{2} \eta^{2}\right)$ and $\zeta^{2}=1 /\left(2 \eta^{2} \beta\right)$ while the GL parameter is $\kappa^{2}=\beta /\left(2 \pi q^{2}\right)$.

For arbitrary $\kappa$ the minimal energy configurations satisfy the second order Euler Lagrange equations, 


$$
\begin{gathered}
D_{i} D_{i} \phi=-2 \frac{\delta V}{\delta \phi^{*}} \\
\frac{1}{4 \pi} \partial_{i} F_{i j}=-j_{j}=-q / 2 i\left(\phi^{*} D_{j} \phi-\phi D_{j} \phi^{*}\right)
\end{gathered}
$$

Using the identity

$$
\frac{1}{4}\left|D_{i} \phi \mp i \epsilon_{i j} D_{j} \phi\right|^{2}=\frac{1}{2}\left|D_{i} \phi\right|^{2} \pm \frac{1}{2 q} \epsilon_{i j} \partial_{i} J_{j} \pm \frac{q}{2} B\left|\phi^{2}\right|
$$

the energy at the self-dual point can be re-written as

$$
E=\int d^{2} x\left(\frac{1}{16 \pi}\left(F_{i j} \pm q 2 \pi \epsilon_{i j}\left(|\phi|^{2}-\eta^{2}\right)\right)^{2}+\frac{1}{4}\left|D_{i} \phi \mp i \epsilon_{i j} D_{j} \phi\right|^{2} \mp \frac{1}{2 q} \epsilon_{i j} \partial_{i} J_{j}\right) \mp \frac{q \eta^{2}}{2} \Phi .
$$

where $\Phi=\int_{\Omega} d^{2} x B=\oint_{\partial \Omega} \vec{A} \cdot d \vec{x}$ is the total magnetic flux through the sample. Assuming that the current is zero at the boundary, a lower bound for the energy is obtained,

$$
E \geq\left|\frac{q \eta^{2}}{2} \Phi\right|
$$

Energy configurations saturating the bound must satisfy the self-dual or Bogomol'nyi equations,

$$
\begin{gathered}
F_{i j} \pm 2 \pi q \epsilon_{i j}\left(|\phi|^{2}-\eta^{2}\right)=0, \\
D_{i} \phi \mp i \epsilon_{i j} D_{j} \phi=0 .
\end{gathered}
$$

In the plane these equations are totally equivalent to the Euler Lagrange equations. To obtain finite energy conditions, one has to demand

$$
\lim _{\rho \rightarrow \infty} D_{i} \Phi=0 \quad \lim _{\rho \rightarrow \infty}|\Phi|^{2}=\eta^{2}
$$

which in turns implies that

$$
\lim _{\rho \rightarrow \infty} J_{i}=0
$$

On the other hand, writing $\phi=|\phi| e^{i \chi}$, the current takes the form:

$$
J_{i}=q|\phi|^{2}\left(\partial_{i} \chi-q A_{i}\right) .
$$

As $\phi$ is a single valued field, the phase $\chi(\rho, \theta)$ must satisfy

$$
\chi(\rho, 2 \pi)-\chi(\rho, 0)=2 \pi n,
$$

with $n$ an integer. This condition together with (11) implies that the total flux has to be an integer multiple of the quantum of the flux $\Phi_{0}=2 \pi / q$

$$
\Phi=\int_{\partial \Omega} A_{i} d x^{i}=\frac{1}{q} \int_{\partial \Omega} \partial_{i} \chi d x^{i}=n \Phi_{0},
$$

For each integer $n$, equations (8), (9) admit a family of solutions depending on $2|n|$ parameters that can be identified as the 2 dimensional coordinates of $|n|$ non interacting vortices with flux quantum $\Phi_{0}$ (the upper or lower sign in the equations has to be chosen according to the sign of $n$ )( [7]).

Let us now concentrate to the case of finite geometries with a boundary. When the current is zero at the boundary, the Bogomol'nyi inequality still provides a bound for the energy and the flux is quantized in units of $\Phi_{0}$. However, for a given flux, there is a minimal area of the sample for which the equations admit solutions. Indeed, integrating the first equations we get the inequality: 


$$
\Phi=\int_{\Omega} B=\int_{\Omega} 2 \pi q\left(\eta^{2}-|\phi|^{2}\right) \leq \int_{\Omega} 2 \pi \eta^{2}=\eta^{2} \frac{q}{2} \operatorname{area}(\Omega) .
$$

In the infinite plane the requirement of zero current at the boundary is the natural boundary condition since it is the only way of obtaining finite energy solutions. However there is no compelling reason to do so for a finite region. The appropriate boundary condition is [8],

$$
D_{\perp} \phi=0
$$

Notice that this boundary condition only implies vanishing of the normal component of the current at the boundary. The tangential component is left in principle undetermined. Nevertheless, it is possible to show that for configurations satisfying the self-dual equations, it holds the relation,

$$
J_{i}= \pm \frac{q}{2} \epsilon_{i j} \partial_{j}\left|\phi^{2}\right|
$$

That is, for solutions of the BE equations both components of the current must vanish at the boundary. As discussed above, this implies that the total flux is quantized.

Suppose now that instead of imposing a boundary condition over $J_{\|}$, we fix the total flux $\Phi$. It is clear, that if $\Phi / \Phi_{0}$ is not an integer, a tangential component of the current will be established at the boundary and minimal energy configurations will not be given by solutions to the BE but by solutions to the Euler Lagrange equations. More interestingly, when $\Phi / \Phi_{0}=m$, with $m$ integer, although the BE do admit solutions, they are not the minimal energy configuration. In fact, it is energetically favorable to create $n \leq m$ vortices and to develop a tangential current at the boundary.

Let us illustrate this in a cylindrically symmetric ansatz:

$$
\begin{aligned}
\phi(x) & =f(\rho) e^{i n \theta} \\
A_{\theta}(x) & =A(\rho) \\
A_{\rho}(x) & =0 .
\end{aligned}
$$

Defining dimensionless variables $x(r)=n-q A(r), z(r)=f(r) / \eta, r=(\rho / \lambda)$, the self-dual equations become

$$
\begin{aligned}
x^{\prime} \pm \frac{r}{2}\left(z^{2}-1\right) & =0 \\
z^{\prime} \mp \frac{x z}{r} & =0
\end{aligned}
$$

and the boundary condition (16) translates into a Neumann condition for the order parameter:

$$
z^{\prime}\left(R^{*}\right)=0
$$

where $R^{*}=R / \lambda$. Thus, we see that unless $x\left(R^{*}\right)=0$ (which implies that $\Phi=\oint A=2 \pi n / q$ ) the BE (20) cannot be satisfied at $r=R^{*}$.

Minimal energy solutions are then obtained by solving the second order Euler-Lagrange equations. In our ansatz they read,

$$
\begin{gathered}
\frac{d^{2} x}{d r^{2}}-\frac{1}{r} \frac{d x}{d r}-x z^{2}=0 \\
\frac{d^{2} z}{d r^{2}}+\frac{1}{r} \frac{d z}{d r}-\frac{x^{2} z}{r^{2}}+\kappa^{2} z\left(1-z^{2}\right)=0
\end{gathered}
$$

while the energy can be expressed as,

$$
E=\pi \eta^{2} \int_{0}^{R^{*}} r d r\left[\left(\frac{x^{\prime}}{r}\right)^{2}+z^{\prime 2}+\frac{x^{2} z^{2}}{r^{2}}+\kappa^{2}\left(z^{2}-1\right)^{2}\right] .
$$

Regularity of cylindrical coordinates impose conditions at $r=0$, and together with the Neumann condition (21) and the definition of $x\left(R^{*}\right)$ in terms of $n$ and $\Phi$ we have the following set of boundary conditions: 


$$
\begin{array}{ll}
x(0)=n & x\left(R^{*}\right)=n-\frac{\Phi}{\Phi_{0}} \\
z(0)=0 & z^{\prime}\left(R^{*}\right)=0 .
\end{array}
$$

We have analyzed the existence of solutions of the system (22,23,25) by numerical integration. We employed a relaxation method for boundary value problems [9]. In such method, the differential equations are discretized in a convenient mesh and converted to a set of coupled algebraic equations. The system is then solved using Newton's iterative method, starting from an initial guess and improving it iteratively. Given $\Phi$ and $R^{*}$ a solution for each integer $n$ is found corresponding to a local minimum of the energy. Then, the $n$ giving the lowest energy is selected.

In figure 1 we show the solutions corresponding to $\Phi=6 \Phi_{0}, R^{*}=10$. For these values, the minimal energy configuration corresponds to $n=1$. The energy of this solution is $E=3.51 \pi \eta^{2}$. As the external flux is an integer number of flux quanta, the Bogomoln'nyi equations also have solutions for this case with energy $E=6 \pi \eta^{2}$. Thus, the system lowers its energy by allowing a tangential component of the current $J_{\|}\left(R^{*}\right) \neq 0$.

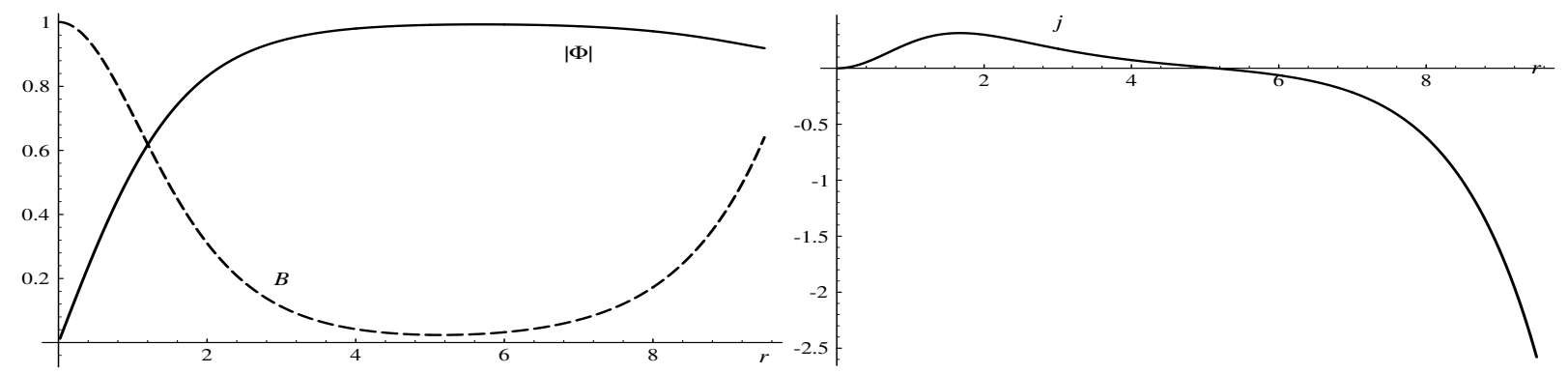

FIG. 1. Vortex solution and angular component the current $j(r)$ for $R=10, n=1$ and $\Phi=6 \Phi_{0}$.

Notice nevertheless that there is a point $R_{0}^{*}$ such that $J\left(R_{0}^{*}\right)=0$. Following reference [4], we will consider the disk as $\Omega=\Omega_{1} \cup \Omega_{2}$ where $\Omega_{1}$, is the inner disk $0 \leq r \leq R_{0}^{*}$ and $\Omega_{2}$, the outer ring $R_{0}^{*} \leq r \leq R^{*}$. We will express the energy as:

$$
E(\Omega)=E\left(\Omega_{1}\right)+E\left(\Omega_{2}\right)
$$

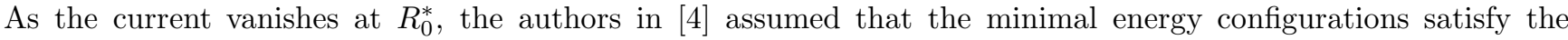
Bogomol'nyi equations in $\Omega_{1}$; however it can be shown that this is not the case. Consider the function $L[r]=$ $z^{\prime}(r) \mp x(r) z(r) / r$, evaluated with the solutions of the Euler-Lagrange equations. If the Bogomol'nyi equations are satisfied on $\Omega_{1}$, then $L[r]=0$ in this region. But, as the current is not zero on the external boundary $r=R^{*}$, the Bogomol'nyi equations are not satisfied on $\Omega_{2}$, and thus $L[r] \neq 0$ on $\Omega_{2}$. Clearly, regularity of the solutions of ordinary differential equations prevents the existence of a function that vanishes in the whole region $0<r<R_{0}^{*}$ but is different from zero for $r>R_{0}^{*}$. Although the solutions of the self-dual equation minimize the energy on the internal region $\Omega_{1}$, any regular extension of the solution to the whole disk will not minimize the total energy.

Having said this we should notice that even though the self-dual solutions are not exact solutions in the inner disk $\Omega_{1}$, they are in fact a very good approximation. A numerical analysis of both solutions shows that for a wide range of parameters, they differ only in around one part in a thousand and the same is true for the energy. Then we will take,

$$
E\left(\Omega_{1}\right) \approx \pi \eta^{2}|n|
$$

Let us now analyze the contribution of the $\Omega_{2}$ region to the energy.

$$
E\left(\Omega_{2}\right)=\pi \eta^{2} \int_{R_{0}^{*}}^{R^{*}} r d r\left[\left(\frac{x^{\prime}}{r}\right)^{2}+z^{\prime 2}+\frac{x^{2} z^{2}}{r^{2}}+\frac{1}{2}\left(z^{2}-1\right)^{2}\right]
$$

We first review the main steps in [4]. There it was assumed that as the fields are concentrated in a region of width of order one from the border, this expression could be approximated as 


$$
\left.E\left(\Omega_{2}\right) \approx \pi \eta^{2} r\left[\left(\frac{x^{\prime}}{r}\right)^{2}+z^{\prime 2}+\frac{x^{2} z^{2}}{r^{2}}+\frac{1}{2}\left(z^{2}-1\right)^{2}\right]\right|_{r=R^{*}} .
$$

Then, the condition $\frac{\delta E}{\delta z}=0$ would give

$$
\frac{x^{2}(R)}{R^{2}}=1-z^{2}
$$

As a next step Akkermans and Mallick neglected the magnetic energy contribution (first term in (29)) arriving to the following expression for the energy,

$$
E\left(\Omega_{2}\right) \approx \pi \eta^{2} R^{*}\left(\frac{x^{2}\left(R^{*}\right)}{R^{* 2}}-\frac{1}{2} \frac{x^{4}\left(R^{*}\right)}{R^{* 4}}\right)
$$

Finally, the authors neglected, in the large $R$ limit, the quartic term in (31) ending with the expression

$$
E\left(\Omega_{2}\right) \approx \pi \eta^{2} \frac{x^{2}\left(R^{*}\right)}{R^{*}}
$$

The reasoning above suffers from two main drawbacks. Although the field $z$ is practically constant, the field $x$ is not, making the approximation of the integral not valid. In fact, our numerical simulation shows that equation (30) is not fulfilled. Second, as shown in fig 2 the magnetic energy is of the same order than the third term in (28).

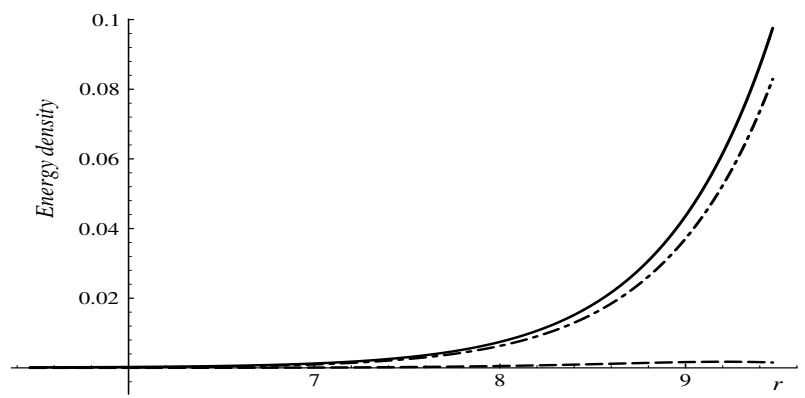

FIG. 2. Energy density contributions near the edge for various terms $\left(R^{*}=10, n=1\right.$ and $\left.\Phi=6 \Phi_{0}\right)$. The filled line corresponds to the term $B^{2}$, the dashed-dotted line corresponds to the $|\phi|^{2}|\nabla \chi-A|^{2}$ term , and the dashed line to the term $|\nabla \Phi|^{2}$. Note that the $B^{2}$ term provides the largest contribution to the energy and thus cannot be neglected.

The correct field distribution in $\Omega_{2}$ can instead be obtained by a simple approximation using

$$
\nu=\frac{\left(n-\frac{\Phi}{\Phi_{0}}\right)}{R^{*}}
$$

as an expansion parameter. Indeed, defining

$$
\tilde{x}=x / \nu
$$

the equations of motion become

$$
\begin{gathered}
\frac{d^{2} \tilde{x}}{d r^{2}}-\frac{1}{r} \frac{d \tilde{x}}{d r}-z^{2} \tilde{x}=0 \\
\frac{d^{2} z}{d r^{2}}+\frac{1}{r} \frac{d z}{d r}-\nu^{2} \frac{\tilde{x}^{2} z}{r^{2}}+\frac{1}{2} z\left(1-z^{2}\right)=0
\end{gathered}
$$


with the boundary conditions

$$
\begin{array}{cc}
\tilde{x}\left(R_{0}^{*}\right)=0 & \tilde{x}\left(R^{*}\right)=R^{*} \\
z\left(R_{0}^{*}\right)=1 & z^{\prime}\left(R^{*}\right)=0
\end{array}
$$

In order to solve these equations, we make an expansion of the form

$$
\begin{aligned}
& \tilde{x}=\tilde{x}_{0}+\nu^{2} \tilde{x}_{2}+O\left(\nu^{4}\right) \\
& z=1+\nu^{2} z_{2}+O\left(\nu^{4}\right)
\end{aligned}
$$

The boundary conditions will be satisfied by the solution $\tilde{x}_{0}$ while homogeneous conditions are valid for $\tilde{x}_{2}$ and $z_{2}$. To lowest order we obtain

$$
\frac{d^{2} \tilde{x}_{0}}{d r^{2}}-\frac{1}{r} \frac{d \tilde{x}_{0}}{d r}-\tilde{x}_{0}=0
$$

with solution

$$
\tilde{x}_{0}(r)=c_{0} r\left[I_{1}(r)-\frac{I_{1}\left(R_{0}^{*}\right)}{K_{1}\left(R_{0}^{*}\right)} K_{1}(r)\right]
$$

Here $c_{0}^{-1}=\left[I_{1}\left(R^{*}\right)-\frac{I_{1}\left(R_{0}^{*}\right)}{K_{1}\left(R_{0}^{*}\right)} K_{1}\left(R^{*}\right)\right]$ and $I_{1}(r), K_{1}(r)$ are Bessel functions.

With these results we can solve the order $\nu^{2}$ equations:

$$
\begin{gathered}
\frac{d^{2} \tilde{x}_{2}}{d r^{2}}-\frac{1}{r} \frac{d \tilde{x}_{2}}{d r}-\tilde{x}_{2}=2 z_{2} \tilde{x}_{0}, \\
\frac{d^{2} z_{2}}{d r^{2}}+\frac{1}{r} \frac{d z_{2}}{d r}-z_{2}=\frac{\tilde{x}_{0}^{2}}{r^{2}}
\end{gathered}
$$

with homogeneous boundary conditions for both functions. Using the Green functions for each equation we obtain the solution

$$
z_{2}(r)=y_{2}(r) \int_{R_{0}^{*}}^{r} \frac{y_{1}\left(r^{\prime}\right) f\left(r^{\prime}\right)}{W_{y_{1}, y_{2}}} d r^{\prime}+y_{1}(r) \int_{r}^{R^{*}} \frac{y_{2}\left(r^{\prime}\right) f\left(r^{\prime}\right)}{W_{y_{1}, y_{2}}} d r^{\prime}
$$

where

$$
\begin{aligned}
y_{1}(r) & =K_{0}(r)-\frac{K_{0}\left(R_{0}^{*}\right)}{I_{0}\left(R_{0}^{*}\right)} I_{0}(r) \\
y_{2}(r) & =K_{0}(r)-\frac{K_{0}^{\prime}\left(R^{*}\right)}{I_{0}^{\prime}\left(R^{*}\right)} I_{0}(r) \\
f(r) & =\frac{\tilde{x}_{0}^{2}}{r^{2}}
\end{aligned}
$$

and $W_{y_{1}, y_{2}}$ is the Wronskian.

The same steps are followed to calculate $\tilde{x}_{2}$ for which we obtain

$$
\tilde{x}_{2}(r)=Y_{2}(r) \int_{R_{0}^{*}}^{r} \frac{Y_{1}\left(r^{\prime}\right) g\left(r^{\prime}\right)}{W_{Y_{1}, Y_{2}}} d r^{\prime}+Y_{1}(r) \int_{r}^{R^{*}} \frac{Y_{2}\left(r^{\prime}\right) g\left(r^{\prime}\right)}{W_{Y_{1}, Y_{2}}} d r^{\prime}
$$

In this case

$$
\begin{aligned}
Y_{1}(r) & =r\left[K_{1}(r)-\frac{K_{1}\left(R_{0}^{*}\right)}{I_{1}\left(R_{0}^{*}\right)} I_{1}(r)\right] \\
Y_{2}(r) & =r\left[K_{1}(r)-\frac{K_{1}\left(R^{*}\right)}{I_{1}\left(R^{*}\right)} I_{1}(r)\right] \\
g(r) & =2 z_{2} \tilde{x}_{0}
\end{aligned}
$$


Having obtained the solutions $\tilde{x}=\tilde{x}_{0}+\nu^{2} \tilde{x}_{2}$ and $z=1+\nu^{2} z_{2}$ we can now consider the expression for the energy in this expansion:

$$
\begin{aligned}
E\left(\Omega_{2}\right) & =\pi \eta^{2} \int_{R_{0}^{*}}^{R^{*}} d r\left\{\frac{\nu^{2}}{r}\left[\left(\frac{d \tilde{x}_{0}}{d r}\right)^{2}+\tilde{x}_{0}^{2}\right]+\nu^{4}\left[r\left(\frac{d z_{2}}{d r}\right)^{2}+\frac{2}{r}\left(\frac{d \tilde{x}_{0}}{d r} \frac{d \tilde{x}_{2}}{d r}+\tilde{x}_{0}^{2} z_{2}+\tilde{x}_{0} \tilde{x}_{2}\right)\right.\right. \\
& \left.\left.+r z_{2}^{2}\right]+O\left(\nu^{6}\right)\right\}
\end{aligned}
$$

Using the equations of motion, the energy takes the form

$$
\frac{E(\Omega)}{\pi \eta^{2}}=n+\nu^{2} \frac{d \tilde{x}_{0}}{d r}\left(R^{*}\right)+\nu^{4} \int_{R_{0}^{*}}^{R^{*}} d r \frac{\tilde{x}_{0}^{2} z_{2}}{r}
$$

The above expression gives us the energy of the vortex configuration up to the order $\nu^{4}$. However an obvious drawback of this expression is the presence of the point $R_{0}^{*}$, which should be located numerically, and then preventing any analytical predictability power of the equation. Nevertheless we can convince ourselves that the point $R_{0}^{*}$ can be shrunk to zero. The reason is that at leading order we can approximate the whole solution as a superposition of a Bogolmo'nyi vortex and a vortex concentrated at the boundary. Because both kind of solutions are exponentially small in complementary regions, any contribution from the non-linearity of the equations is exponentially suppressed. In fact, a simple plot of the solutions (41,44, 46) shows that the solution with $R_{0}^{*}=0$ only differs in about $10^{-3}$ with the one with non-zero $R_{0}^{*}$. Therefore, we will take $R_{0}^{*}=0$.

In this case the energy can be found by a simple numerical integration. Notice that the integrals only depends on $R^{*}$ and not on $\Phi$ or $n$. The result can be compared with the obtained in reference [ 4 . In the following table we show a comparison between the energy values obtained from our approximate equation (50), the ones obtained from the expression of reference and [4] and the exact ones, corresponding to vortex solutions with $R=10$ and $n=1$. We see that even for big values of $\nu$, our approximate equation gives a result in excellent agreement with the exact ones.

\begin{tabular}{|c|c|c|c|}
\hline$\Phi$ & $\begin{array}{c}\text { Energy } \\
\text { from (50) }\end{array}$ & $\begin{array}{c}\text { Energy } \\
\text { of Ref. }\end{array}$ & $\begin{array}{c}\text { Exact } \\
\text { energy }\end{array}$ \\
\hline 1.0 & 1.0 & 1.0 & 1.0 \\
3.0 & 1.42 & 1.4 & 1.42 \\
5.0 & 2.64 & 2.6 & 2.64 \\
8.0 & 5.78 & 5.9 & 5.79 \\
12.0 & 11.51 & 13.1 & 11.27 \\
18.0 & 18.81 & 29.9 & 18.0 \\
\hline
\end{tabular}

Furthermore we can find a large $R$ expansion of equation (50). Using the asymptotic expansion of the Bessel functions, the first term in (50) has the asymptotic form $\nu^{2}\left(R^{*}+0.5+O\left(1 / R^{*}\right)\right)$. The coefficient of $\nu^{4}$ term was found by a numerical fit. The resulting approximate expression for the energy is

$$
\frac{E}{\pi \eta^{2}}=n+\nu^{2} R^{*} a\left(R^{*}\right)-\nu^{4} R^{*} b\left(R^{*}\right)+O\left(1 / R^{*}, \nu^{6}\right)
$$

where

$$
a\left(R^{*}\right)=1+1 /\left(2 R^{*}\right), \quad b\left(R^{*}\right)=0.139+0.111 / R^{*}
$$

The most stable configuration corresponds to a vortex solution with vortex number $n$ such that is an absolute minimum of the energy. Equation (51) can be approximately minimized with respect to $n$, with $b\left(R^{*}\right) / a\left(R^{*}\right)$ as the expansion parameter. We find that the vortex number that minimizes the energy is given by: 


$$
n=\left[\frac{\Phi}{\Phi_{0}}-\frac{R^{*}}{2 a}\left(\frac{a^{3}-b}{a^{3}-3 / 2 b}\right)+\frac{1}{2}\right]
$$

where $[x]$ means the integer part of $x$.

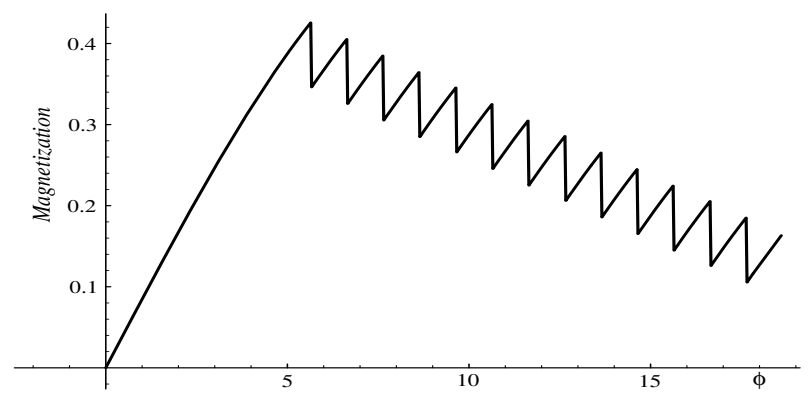

FIG. 3. The magnetization $-M /\left(q \eta^{2}\right)$ as a function of the external flux $\Phi / \Phi_{0}$ for $R^{*}=10$.

The magnetization of the system shown in fig.3 is obtained from the Gibbs free energy, $G=E-2 \pi \eta^{2}\left(\Phi / \Phi_{0}\right)^{2} / R^{* 2}$, as

$$
M=-\frac{\partial G}{\partial \Phi}=-\frac{\pi \eta^{2}}{\Phi_{0}}\left(\frac{2 a\left(R^{*}\right)}{R^{*}}\left(\frac{\Phi}{\Phi_{0}}-n\right)-\frac{4 b\left(R^{*}\right)}{R^{* 3}}\left(\frac{\Phi}{\Phi_{0}}-n\right)^{3}-4 \frac{\Phi}{\Phi_{0}} \frac{1}{R^{* 2}}\right)
$$

where $n$ is given in equation (53).

In this work we have analyzed the existence of vortex solutions of the Ginzburg Landau theory at the self dual point $\kappa^{2}=1 / 2$ for a two dimensional disk of finite radius. Our original aim was to pursue further the interesting proposal made by Akkermans and Mallick [4] of exploiting the properties of the Bogomol'nyi equations for the study of the vortices at the self-dual point. Unfortunately our numerical study revealed that some of the assumptions made there are not entirely correct.

We have shown that the minimal energy configurations do not satisfy the Bogomol'nyi equations in the inner disk. Nevertheless they provide a very good approximation to the actual solutions. Concerning the behaviour of the fields in the outer ring we have provided a simple analytical approximation scheme which do conform the numerical simulation and allows to obtain a selection rule for the number of vortices as a function of the external flux.

[1] E.B.Bogomol'nyi, Sov.J.Nucl.Phys. 24449 (1976), (Yad.Fiz 24861 (1976); H.de Vega and F.A.Schaposnik, Phys.RevD14 $1100(1976)$

[2] J.L.Harden and V.Arp, Cryogenics 3105 (1963)

[3] L.Alvarez Gaumé y F.Zamora, "Duality in Quantum Field Theory (and String Theory)", in Trends in Theoretical Physics, AIP conference proceedings 419, (ed by H.Falomir et al), (1997).

[4] E.Akkermans and K.Mallick, cond-mat0001219 (unpublished); J.Phys.A 327133 (1999); cond-mat 9907441 (unpublished)

[5] A.K.Geim et al, Nature 390259 (1997), ibid 396144 (1998).

[6] F.M.Peeters, V.A.Schweigert,B.J.Baelus and P.S.Deo, "Vortex Matter in Mesoscopic Superconducting Disks and Rings", cond-mat 9910172 and references therein.

[7] "Vortices and monopoles. Structure of static gauge theories".by A. Jaffe, C. Taubes (Harvard U.). 1980. Boston, Usa: Birkhaeuser (1980) 287 P.(Progress In Physics, 2).

[8] D.Saint-James, E.Thomas and G.Sarma. "Type II Superconductivity", Pergamon Press (1969).

[9] W.H. Press, S.A. Teukolsky and W.T. Vetterling, Numerical Recipes: The art of Scientific Computing, Cambridge University Press, (1992). 